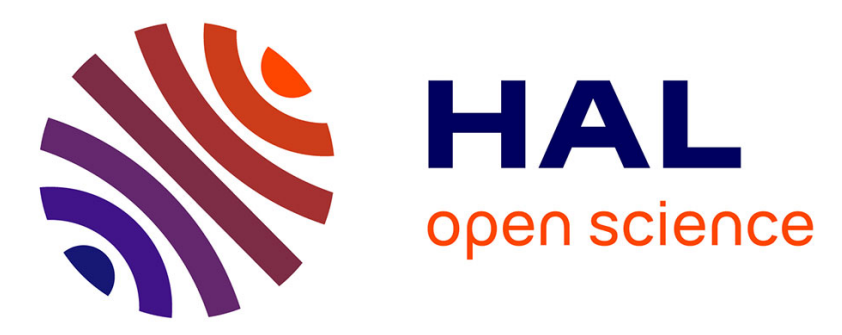

\title{
Etude expérimentale de la zone pariétale d'un écoulement turbulent instationnaire en conduite bidimensionnelle
}

\author{
J. Tartarin
}

\section{- To cite this version:}

J. Tartarin. Etude expérimentale de la zone pariétale d'un écoulement turbulent instationnaire en conduite bidimensionnelle. Revue de Physique Appliquée, 1983, 18 (8), pp.495-505. 10.1051/rphysap:01983001808049500 . jpa-00245111

\section{HAL Id: jpa-00245111 https://hal.science/jpa-00245111}

Submitted on 1 Jan 1983

HAL is a multi-disciplinary open access archive for the deposit and dissemination of scientific research documents, whether they are published or not. The documents may come from teaching and research institutions in France or abroad, or from public or private research centers.
L'archive ouverte pluridisciplinaire HAL, est destinée au dépôt et à la diffusion de documents scientifiques de niveau recherche, publiés ou non, émanant des établissements d'enseignement et de recherche français ou étrangers, des laboratoires publics ou privés. 


\title{
Etude expérimentale de la zone pariétale d'un écoulement turbulent instationnaire en conduite bidimensionnelle
}

\author{
J. Tartarin \\ Laboratoire d'Etudes Aérodynamiques, \\ 40, avenue du Recteur Pineau, 86022 Poitiers, France
}

(Reçu le 23 novembre 1982, révisé le 5 avril 1983, accepté le 19 avril 1983)

\begin{abstract}
Résumé. - L'étude expérimentale de la zone pariétale d'un écoulement bidimensionnel turbulent dont le débit est perturbé périodiquement a été menée en examinant plus particulièrement l'influence de la fréquence de perturbation lorsque celle-ci est de l'ordre de grandeur de la fréquence caractéristique d'apparition des bouffées turbulentes. Des variations notables dans l'évolution des profils des champs périodiques de vitesse et de turbulence très près de la paroi $\left(y^{+}<25\right)$ nous ont conduit à proposer une hypothèse concernant la phénoménologie de la turbulence dans cette zone.
\end{abstract}

\begin{abstract}
Experimental study in the near wall region of a two-dimensionnal turbulent flow, with oscillating perturbation, was performed with particular attention focused on the influence of the imposed frequency, when close to the burst frequency. Noticeable changes in the turbulence and velocity periodic fields profiles in the viscous and buffer layers $\left(y^{+}<25\right)$ led us to propose an assumption concerning the behaviour of the turbulence in that region.
\end{abstract}

\section{Introduction.}

Les recherches consacrées aux écoulements turbulents instationnaires ont suivi au cours des dix dernières années deux axes majeurs : l'un, théorique, a contribué à développer les modèles mathématiques permettant la prévision numérique des grandeurs caractéristiques liées à ce type d'écoulement; le second, expérimental, a mis en évidence les difficultés à obtenir, au laboratoire, des écoulements instationnaires turbulents dont tous les paramètres seraient accessibles et contrôlables dans une large gamme. Ceci explique la dispersion et la disparité des résultats obtenus jusqu'alors et l'insuffisance de ceux-ci quant à fournir des données « universelles » permettant, par exemple, la fermeture des équations modélisées numériquement.

Pour notre part, nous avons abordé cette classe d'écoulements en explorant expérimentalement la zone proche de la paroi d'un canal bidimensionnel dont le débit est perturbé périodiquement. Plus particulièrement, nous avons examiné le comportement des grandeurs caractéristiques du champ de vitesse dans ce domaine, en fonction de la fréquence de la perturbation, dans une gamme réduite autour de la fréquence caractéristique d'apparition des bouffées turbulentes.

\section{Rappels bibliographiques.}

L'idée directrice des mesures entreprises s'appuie sur une étude bibliographique des divers résultats obtenus d'une part dans le domaine des écoulements turbulents cisaillés instationnaires et, d'autre part, dans celui des phénomènes d'apparition des bouffées turbulentes en écoulements stationnaires.

2.1 A PROPOS DES BOUFFÉES TURBULENTES. - Il est couramment admis [1-11] en écoulements turbulents de type couche limite que des structures assez régulières, ayant l'aspect de "filets ", apparaissent très près de la paroi $\left(y^{+}<10\right)$. Ces filets, dont la vitesse est inférieure à la vitesse locale, deviennent instables, quittent la paroi, sont rompus et éjectés brutalement. Ce processus d'éjection, désigné par «bouffées turbulentes " ( "bursts », dans la littérature anglosaxonne) est lié à l'arrivée de fluide à vitesse élevée, qui pénètre de haut en bas jusque dans la sous-couche visqueuse. L'échantillonnage conditionnel permet de mettre en évidence les quatre séquences suivantes :

$$
\begin{array}{ll}
u^{\prime}<0, v^{\prime}>0 & \text { (éjection de fluide) } \\
u^{\prime}>0, v^{\prime}<0 & \text { (apport de fluide) } \\
u^{\prime}>0, v^{\prime}>0 & \\
u^{\prime}<0, v^{\prime}<0 &
\end{array}
$$


Les deux premières séquences (donnant un produit négatif $u^{\prime} v^{\prime}<0$, comme les tensions de Reynolds) sont prépondérantes dans la couche limite. Près de la paroi $\left(y^{+}<15\right)$, la séquence "apport de fluide " domine, tandis qu'au-delà $\left(y^{+}>15\right)$ on constate la situation inverse. L'échelle de temps $\mathrm{du}$ processus d'apparition des bouffées turbulentes (notée : $N_{\mathrm{b}}$ ) est liée aux paramètres globaux de l'écoulement $[6,7,9,28]$ : vitesse extérieure $\left(\bar{U}_{\mathrm{e}}\right)$ et épaisseur de couche limite $(\delta)$ dans le cas d'écoulements libres; vitesse moyenne sur l'axe $\left(\bar{U}_{\mathrm{c}}\right)$ et épaisseur du canal $(2 \mathrm{H})$ dans le cas d'écoulements confinés. Pour fixer un ordre de grandeur, les valeurs généralement admises sont respectivement :

$$
N_{\mathrm{b}}=\frac{\bar{U}_{\mathrm{e}}}{5 \delta} \text { et } N_{\mathrm{b}}=\frac{\bar{U}_{\mathrm{c}}}{5 H} .
$$

Ces valeurs, qu'il n'est cependant pas possible de considérer comme des constantes " universelles » [35], nous serviront de référence dans la suite de l'exposé pour définir un paramètre adimensionnel pour la classification des écoulements turbulents perturbés périodiquement.

2.2 A PROPOS DES ÉCOULEMENTS PERTURBÉS PÉRIODIQUEMENT. - La plupart des études expérimentales entreprises concernent les écoulements de couche limite turbulente perturbée périodiquement [19-25]. Souvent, pour des raisons technologiques, la fréquence de perturbation est unique, ou fixée arbitrairement dans le domaine "basses fréquences" afin d'obtenir des amplitudes de perturbation suffisantes. Les écoulements turbulents établis en canal bidimensionnel ou en conduite cylindrique ont été abordés de façons très disparates : écoulements pulsés [13,14, 15, 17, 27], parois ondulantes [18], ruban vibrant près de la paroi [12] et ont conduit à des résultats parfois contradictoires.

L. W. Carr [26] a entrepris un recensement exhaustif de ces types d'écoulements dont la publication récente nous a conforté dans les impératifs que nous nous étions fixés a priori au début de notre étude : étudier expérimentalement un écoulement bidimensionnel turbulent instationnaire tel que :

a) la perturbation soit périodique (pratiquement sinusoïdal dans l'axe de la soufflerie, quelle que soit la fréquence),

b) le domaine de fréquences de cette perturbation soit restreint et centré sur la fréquence $N_{\mathrm{b}}$,

c) l'amplitude de perturbation soit faible (de l'ordre de $5 \%$, relativement à la vitesse moyenne sur l'axe de la soufflerie) mais éventuellement variable,

d) la zone de paroi soit le domaine d'investigation privilégiée $\left(3<y^{+}<250\right)$, compte tenu des phénomènes turbulents notables dont elle est le siège. En particulier, les avis convergent pour admettre que la structure de la turbulence n'est affectée que dans la zone très proche de la paroi où peuvent interagir la couche limite et la couche de Stokes.

Les caractéristiques de notre écoulement (cf. chapitre 3.3) permettent de le situer sur le graphique publié par L. W. Carr [26]. Nous avons également noté sur la figure 1 le domaine d'études des travaux de Binder et Kueny [14, 27], non recensés. Ce graphique montre que la confrontation de nos résultats à ceux de Acharya et Reynolds [13] et Binder et Kueny [14, 27] permettra des conclusions intéressantes.

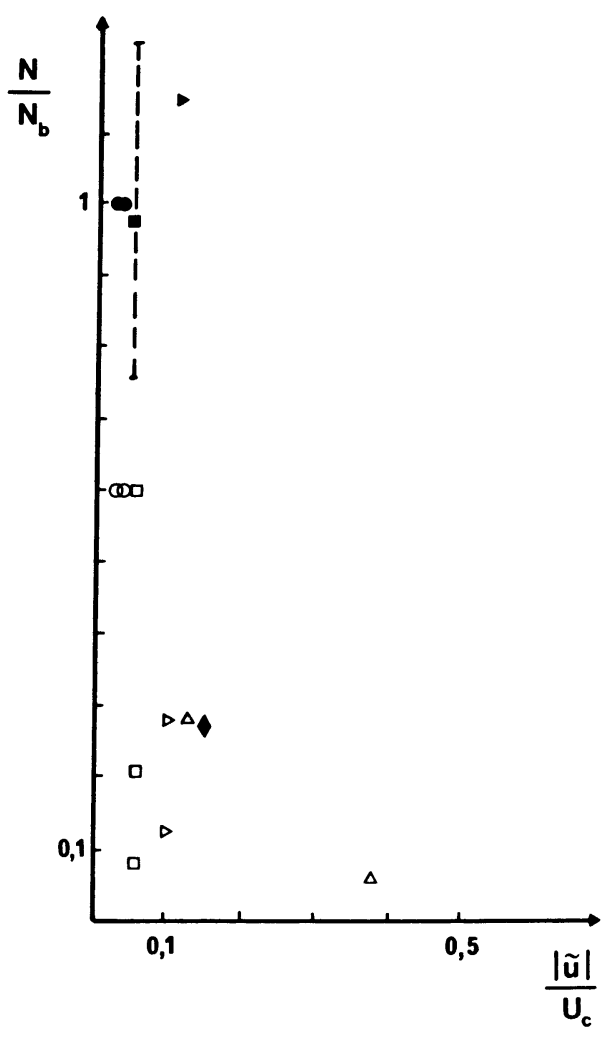

Fig. 1. - Domaine d'expérimentation d'écoulements turbulents instationnaires (d'après L. W. Carr [26]). Acharya et Reynolds [13], $\square \square$ Binder et Kueny [14], $\triangle$ Cousteix et autres $[19,20], \triangleright$ Parikh et autres [23], $\diamond$ Ramaprian et Tu [15], - . Tartarin.

[Field of investigation on some unsteady turbulent flows (from L. W. Carr [26]).]

\section{Rappels théoriques.}

3.1 CONCEPT DE LA « DÉCOMPOSITION TRIPLE ». Hussain et Reynolds [12] ont montré que lorsqu'on imposait une perturbation périodique à un écoulement turbulent, on introduisait une composante périodique supplémentaire aux champs de vitesse et de pression. Toute variable instantanée $f\left(x_{i}, t\right)$ peut donc être décomposée en trois contributions : " moyenne", "périodique » et " turbulente » tel que :

$$
f\left(x_{i}, t\right)=\bar{f}\left(x_{i}\right)+\tilde{f}\left(x_{i}, t\right)+f^{\prime}\left(x_{i}, t\right) .
$$


Ces contributions sont accessibles expérimentalement par un échantillonnage conditionnel permettant d'obtenir une "moyenne en phase ": $\left\langle f\left(x_{i}, t\right)\right\rangle$

$$
\left\langle f\left(x_{i}, t\right)\right\rangle=\bar{f}\left(x_{i}\right)+\tilde{f}\left(x_{i}, t\right) .
$$

En pratique, l'échantillonnage conditionnel d'un signal périodique permet d'obtenir la moyenne en phase ainsi :

$$
\left\langle f\left(x_{i}, \phi_{j}\right)\right\rangle=\lim _{N \rightarrow \infty} \frac{1}{N} \sum_{n=0}^{N} f\left(x_{i}, \phi_{j}+2 n \pi\right) .
$$

Dans notre expérimentation, l'acquisition directe des données portait sur 4000 périodes $(N)$, divisées en $32(j)$ phases égales (la phase origine étant arbitraire mais immuable lors des campagnes de mesures).

Les trois contributions définies ci-dessus sont obtenues ainsi :

$$
\begin{aligned}
\bar{f}\left(x_{i}\right) & =\sum_{j=1}^{32}\left\langle f\left(x_{i}, \phi_{j}\right)\right\rangle . \\
\tilde{f}\left(x_{i}, \phi_{j}\right) & =\left\langle f\left(x_{i}, \phi_{j}\right)\right\rangle-\bar{f}\left(x_{i}\right) . \\
f^{\prime}\left(x_{i}, \phi_{j}\right) & =f\left(x_{i}, \phi_{j}\right)-\left\langle f\left(x_{i}, \phi_{j}\right)\right\rangle .
\end{aligned}
$$

3. 2 Equations De Navier-Stokes. - Les équations de continuité et de Navier-Stokes pour un fluide incompressible à propriétés physiques constantes s'écrivent sous forme adimensionnelle ainsi :

$$
\begin{aligned}
\frac{\partial u_{i}}{\partial x_{i}} & =0 \\
\frac{\partial u_{i}}{\partial t}+u_{j} \frac{\partial u_{i}}{\partial x_{j}} & =-\frac{\partial p}{\partial x_{i}}+\frac{1}{R e} \frac{\partial^{2} u_{i}}{\partial x_{j} \partial x_{j}}
\end{aligned}
$$

Les références sont : $\bar{u}_{\mathrm{c}}$, la vitesse moyenne sur l'axe de la veine; $H$, la demi-hauteur du canal; $v$, la viscosité cinématique et $H / \bar{u}_{\mathrm{c}}$ l'échelle de temps.

Compte tenu de la décomposition triple définie ci-dessus, l'équation de continuité (7) devient :

$$
\frac{\partial \bar{u}_{i}}{\partial x_{i}}=\frac{\partial u_{i}^{\prime}}{\partial x_{i}}=\frac{\partial \tilde{u}_{i}}{\partial x_{i}}=0
$$

La moyenne en phase de l'équation (8) permet d'accéder aux équations générales pour le champ moyen :

$$
\begin{array}{r}
\bar{u}_{j} \frac{\partial \bar{u}_{i}}{\partial x_{j}}=-\frac{\partial \bar{p}}{\partial x_{i}}+\frac{1}{\operatorname{Re}} \frac{\partial^{2} \bar{u}_{i}}{\partial x_{j} \partial x_{j}}-\frac{\partial}{\partial x_{j}} \overline{\left(\overline{u_{i}^{\prime} u_{j}^{\prime}}\right)-} \\
\frac{\partial}{\partial x_{j}}\left(\overline{\tilde{u}_{i} \tilde{u}_{j}}\right) .
\end{array}
$$

le champ périodique :

$$
\begin{aligned}
\frac{\partial \tilde{u}_{i}}{\partial t}+\bar{u}_{j} \frac{\partial \tilde{u}_{i}}{\partial x_{j}} & +\tilde{u}_{j} \frac{\partial \bar{u}_{i}}{\partial x_{j}}= \\
& -\frac{\partial \tilde{p}}{\partial x_{i}}+\frac{1}{\operatorname{Re}} \frac{\partial^{2} u_{i}}{\partial x_{j} \partial x_{j}}+\frac{\partial}{\partial x_{j}}\left(\overline{\tilde{u}_{i} \tilde{u}_{j}}-\tilde{u}_{i} \tilde{u}_{j}\right) \\
& -\frac{\partial}{\partial x_{j}}\left(\left\langle u_{i}^{\prime} u_{j}^{\prime}\right\rangle-\overline{u_{i}^{\prime} u_{j}^{\prime}}\right)
\end{aligned}
$$

La bidimensionnalité de l'écoulement étudié expérimentalement $(\bar{u}=\bar{u}(y), \bar{v}=\bar{w}=0)$, perturbé périodiquement $(\tilde{u}=\tilde{u}(y, t), \tilde{v}=\tilde{w}=0)$ conduit à des équations simplifiées :

3.2.1. Pour le champ « moyen».

$$
\begin{gathered}
\frac{\partial \bar{u}}{\partial x}=0 . \\
0=-\frac{\partial \bar{p}}{\partial x}+\frac{1}{R e} \frac{\partial^{2} \bar{u}}{\partial y^{2}}-\frac{\partial}{\partial y}\left(\overline{u^{\prime} v^{\prime}}\right),
\end{gathered}
$$

- conditions aux limites associées :

- à la paroi $(y=0)$

$$
\bar{u}=0
$$

- sur l'axe du canal $(y=H)$

$$
\left\{\begin{array}{l}
\frac{\partial \bar{u}}{\partial y}=0 \\
\overline{u^{\prime} v^{\prime}}=\overline{r_{12}}=0 .
\end{array}\right.
$$

\subsubsection{Pour le champ " périodique ».}

$\frac{\partial \tilde{u}}{\partial x}=0$.

$$
\begin{aligned}
\frac{\partial \tilde{u}}{\partial t} & =-\frac{\partial \tilde{p}}{\partial x}+\frac{1}{R e} \frac{\partial^{2} \tilde{u}}{\partial y^{2}}-\frac{\partial}{\partial y}\left(\left\langle u^{\prime} v^{\prime}\right\rangle-\overline{u^{\prime} v^{\prime}}\right) \\
0 & =-\frac{\partial \tilde{p}}{\partial y}-\frac{\partial}{\partial y}\left(\left\langle v^{\prime} v^{\prime}\right\rangle-\overline{v^{\prime 2}}\right)
\end{aligned}
$$

par analogie avec (5) nous noterons :

$$
\begin{aligned}
& \left\langle u^{\prime} u^{\prime}\right\rangle-\overline{u^{\prime 2}}=\tilde{r}_{11} \\
& \left\langle u^{\prime} v^{\prime}\right\rangle-\overline{u^{\prime} v^{\prime}}=\tilde{r}_{12}, \text { etc... }
\end{aligned}
$$

- conditions aux limites associées :

- à la paroi $(y=0)$

$$
\left\{\begin{array}{l}
\tilde{u}=\tilde{v}=\tilde{w}=0 \\
\tilde{r}_{12}=0
\end{array}\right.
$$

- sur l'axe du canal $(y=H)$

$$
\left\{\begin{array}{l}
\frac{\partial \tilde{u}}{y}=\frac{\partial \tilde{r}_{11}}{y}=\frac{\partial \tilde{r}_{22}}{y}=0 \\
\bar{r}_{12}=\tilde{r}_{12}=0 .
\end{array}\right.
$$

Les équations au second ordre deviennent très vite 
complexes et montrent que la modélisation de tels écoulements est délicate. A titre d'exemple, l'équation régissant la réponse périodique $\tilde{r}_{11}$ des fluctuations turbulentes à la perturbation s'écrit :

$$
\begin{aligned}
\left.\frac{\partial \tilde{r}_{11}}{\partial t}=-2 \tilde{r}_{12} \frac{\partial \bar{u}}{\partial y}-2 \overline{u^{\prime} v^{\prime}} \frac{\partial \tilde{u}}{\partial y}+\frac{\partial}{\partial y} \overline{\left(u^{\prime 2} v^{\prime}\right.}-\left\langle u^{\prime 2} v^{\prime}\right\rangle\right)-2\left\langle u^{\prime} \frac{\partial p^{\prime}}{\partial x}\right\rangle+ & \\
& +2 \overline{u^{\prime} \frac{\partial p^{\prime}}{\partial x}}+\frac{1}{\operatorname{Re}}\left[\frac{\partial^{2} \tilde{r}_{11}}{\partial y^{2}}-2\left\langle\left(\frac{\partial u^{\prime}}{\partial y}\right)^{2}\right\rangle+2\left(\frac{\partial u^{\prime}}{\partial y}\right)^{2}\right] .
\end{aligned}
$$

\section{Moyens expérimentaux.}

4.1 LA SOUFFLERIE. - La soufflerie est un canal aérothermique dont les dimensions sont :

- longueur $L=12,50 \mathrm{~m}$

- largeur $l=1,25 \mathrm{~m}$

- hauteur $2 H=0,1245 \mathrm{~m}$.

Ces dimensions laissent prévoir un écoulement turbulent établi bidimensionnel dans une large zone médiane. Ceci a été vérifié pour ce canal par de précédents expérimentateurs [31].

L'aspiration se fait au travers d'une tuyère sonique de type " Laval » que nous avons modifiée de sorte à obtenir une perturbation périodique du débit. Le montage adopté comporte un système d'ailette, placé perpendiculairement au col de la tuyère et entraîné par un moteur régulé par un dispositif tachymétrique. La fréquence de la perturbation peut ainsi varier de 5 à $100 \mathrm{~Hz}$, à $0,2 \%$ près. Sur l'arbre du moteur est fixé un disque muni de fentes; une cellule photoélectrique permet d'une parț de connaître la fréquence de la perturbation (par l'intermédiaire d'une périodemètre) et d'autre part de commander le début de chaque période d'acquisition. Une seconde barrière optique permet la synchronisation des 32 acquisitions par période. En fonction des dimensions de l'ailette, la perturbation de la vitesse axiale dans le canal a une amplitude pouvant varier de 3 à $8 \%$.

4.2 MÉTHOdeS DE MESURES. - L'appareillage mis en œuvre est classique : sonde simple à fil chaud (diamètre de 5 microns), chaîne anémométrique " DISA » (anémomètre et linéarisateur). L'encombrement d'une sonde croisée en $\mathrm{X}$ nous a conduit, dans un premier temps, à nous limiter aux mesures des vitesses longitudinales, étant donné l'extrême intérêt de la zone très proche de la paroi $\left(y^{+}<25\right)$. Les étalonnages préalables (avec et sans linéarisateur) et les mesures de vitesse moyenne réalisées au tube de Pitot dans l'axe du canal ont permis de vérifier la reproductibilité des valeurs absolues des grandeurs de référence : vitesse sur l'axe $\left(\bar{U}_{\mathrm{c}}\right)$, vitesse de frottement $\left(\bar{u}_{\tau}\right)$ déduite des mesures de gradients de pression [31].

\section{3 Caractéristiques de l'ÉCoulement.}

- Vitesse moyenne sur l'axe du canal à la section de mesure choisie :

$$
\bar{U}_{\mathrm{c}}=7,80 \mathrm{~m} / \mathrm{s} .
$$

- Nombre de Reynolds :

$$
\operatorname{Re}=\frac{\bar{U}_{\mathrm{c}} H}{v}=31300 \text {. }
$$

- Amplitude relative de la composante périodique de la vitesse sur l'axe du canal :

$$
\varepsilon=\frac{\left|\tilde{U}_{\mathrm{c}}\right|}{\bar{U}_{\mathrm{c}}}=5,5 \%
$$

- Vitesse de frottement pariétal :

$$
\bar{u}_{\tau}=0,35 \mathrm{~m} / \mathrm{s} \text {. }
$$

- Echelle de longueur dans la sous-couche visqueuse :

$$
y_{\tau}=\frac{v}{u_{\tau}} \cong 0,044 \mathrm{~mm}
$$

- Ordre de grandeur de l'épaisseur de la souscouche visqueuse est : $0,25 \mathrm{~mm}$.

- Fréquence de la perturbation imposée (par pas de 0,5 ou $1 \mathrm{~Hz}$ )

$$
19 \leqq N \leqq 32 \mathrm{~Hz} .
$$

- Fréquence caractéristique d'apparition des bouffées turbulentes :

$$
N_{\mathrm{b}}=\frac{U_{\mathrm{c}}}{5 H} \cong 25 \mathrm{~Hz}
$$

- Epaisseur de la couche limite de "Stokes», associée au champ de vitesse périodique :

$$
\delta_{\mathrm{A}}=\left(\frac{2 v}{\omega}\right)^{1 / 2} \cong 0,44 \mathrm{~mm}, \quad(\grave{\mathrm{a}} N=25 \mathrm{~Hz}) \text {. }
$$


- Paramètre adimensionnel traduisant le rapport de l'épaisseur de la couche limite de Stokes et de l'échelle de longueur de la sous-couche :

$$
\delta_{\mathrm{A}}^{+}=\frac{\delta_{\mathrm{A}}}{y_{\tau}} \cong 10, \quad(\grave{\mathrm{a}} N=25 \mathrm{~Hz}) .
$$

4. 4 INCERTITUDES EXPÉRIMENTALES. - Dans ce type d'écoulements, l'expérimentateur est confronté aux problèmes posés par les vibrations inéluctables de la structure de la soufflerie. En particulier, des déflections périodiques des plaques constituant les parois du canal sont engendrées par l'oscillation de la pression. Des mesures de vibration (avec accéléromètre et amplificateur de charge Bruel et Kjaer) ont permis de mettre en évidence une fréquence de résonance très pointue autour de $11,7 \mathrm{~Hz}$ (proche de la fréquence de résonance acoustique du canal qui serait de l'ordre de $13,6 \mathrm{~Hz}$ en le considérant comme un tuyau semiouvert). A cette fréquence, les amplitudes de vibration au centre de la plaque de la section de mesure sont effectivement très importantes (de l'ordre de $0,1 \mathrm{~mm}$ ).

Cependant, dans le domaine des fréquences explorées $(19$ à $30 \mathrm{~Hz})$, l'amplitude vibration reste pratiquement constante et de l'ordre de $15 \mu \mathrm{m}$ (en particulier, pour les quatre fréquences de perturbation que nous présentons ci-après). Le porte-sonde n'étant pas solidaire des parois ne vibre pas. Pour les mesures proches de la paroi $\left(y^{+}<10\right)$ la sonde est donc sensible à une vitesse " apparente " $\tilde{u}_{\mathrm{p}}$ due à la déflection périodique de la paroi dont on peut estimer l'ordre de grandeur (13 et 14) :

$$
\tilde{u}_{\mathrm{p}} \cong d \cdot \frac{\partial \bar{u}}{\partial y} \text { près de la paroi }, \frac{\partial \bar{u}}{\partial y} \cong \frac{\bar{u}_{\tau}}{y_{\tau}}
$$

et

$$
d=15 \mu \mathrm{m},
$$

d'où :

$$
\tilde{u}_{\mathrm{p}} \cong 0,12 \mathrm{~m} / \mathrm{s}
$$

Cette vitesse apparente est importante relativement aux plus basses vitesses périodiques mesurées près de la paroi $\left(|\tilde{u}|_{\min } \cong 0,25 \mathrm{~m} / \mathrm{s}\right)$, et il convient donc d'être très prudent quant à l'interprétation des résultats. Malgré cela, nous avons vérifié une bonne reproductibilité de nos mesures (en particulier pour $y^{+} \geqq 6$ ), en rigidifiant progressivement nos parois de la section de mesure à l'aide de "verins" munis de pieds en caoutchouc anti-vibratile. Nous estimons ainsi que la crédibilité de nos mesures peut être assurée à partir de $y^{+}=6$.

\section{Résultats expérimentaux.}

5.1 La figure 2 montre le caractère pratiquement sinusoïdal de la contribution périodique $(\widetilde{u})$ de la

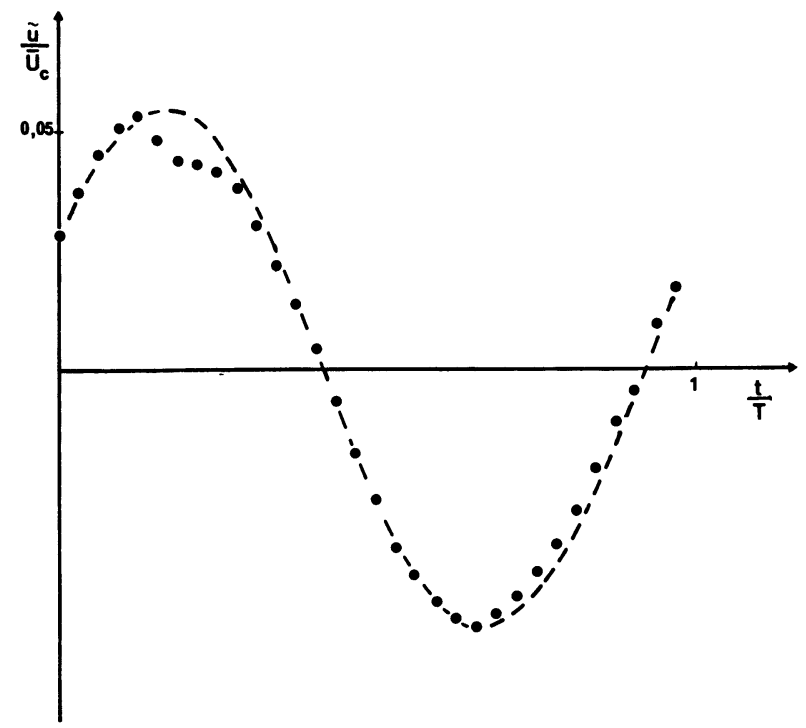

Fig. 2. - Composante périodique de la vitesse longitudinale sur l'axe de la soufflerie $(\bullet)$ et sinusoïde de référence (- - -).

[Periodic velocity component on the axis of the air channel (๑) and reference sine (- - -).]

vitesse longitudinale sur l'axe du canal. Lorsqu'on approche de la paroi apparaît une distorsion inévitable de ce signal, qui reste cependant parfaitement périodique.

5. 2 La figure 3 représente la réponse de la fluctuation turbulente à la perturbation de l'écoulement. Sur l'axe de la soufflerie, la moyenne en phase de la composante turbulente de la vitesse longitudinale présente un caractère sinusoidal distordu. On observe entre les courbes 2 et 3 un déphasage de l'ordre de $\pi / 4$. Près de

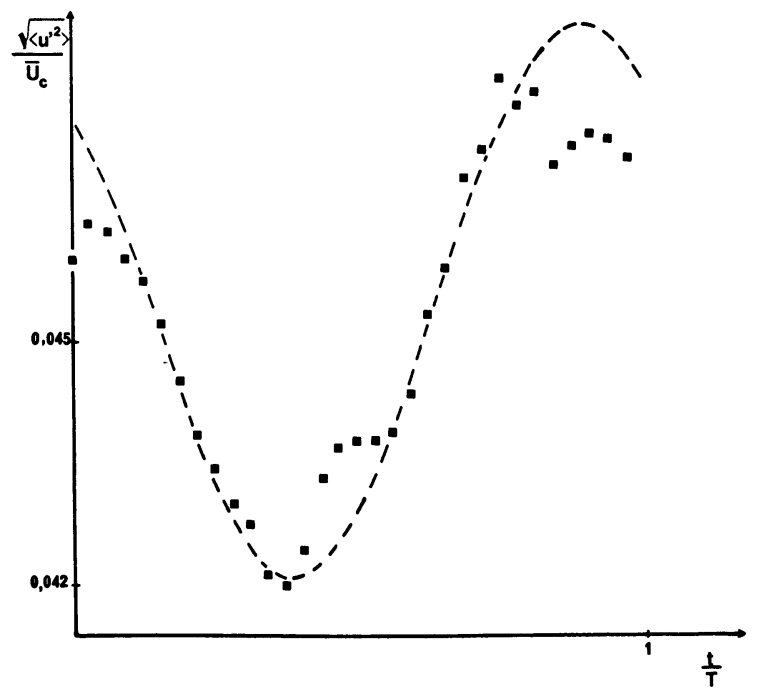

Fig. 3. - Moyenne en phase de l'intensité de turbulence sur l'axe de la soufflerie $(\square)$ et sinusoïde de référence (- - -).

[Ensemble averaged turbulent intensity on the axis of the air channel. ( $\square$ ) and reference sine (- - -).] 
la paroi, le caractère sinusoïdal disparaît presque totalement pour $10<y^{+}<30$, mais réapparaît pour $y^{+}<10$. Ces premières observations montrent qualitativement que la zone intermédiaire (" buffer layer ») est le domaine d'une activité turbulente intense qui masque la contribution périodique du signal turbulent.

5.3 Les profils de vitesse moyenne $(\bar{u})$ dans la zone pariétale montrent une bonne concordance avec ceux obtenus en canal bidimensionnel par d'autres expérimentateurs (Comte-Bellot en stationnaire [32], Achaya et Reynolds en instationnaire [13]) (cf. Fig. 4). La figure 5 met en évidence les différentes zones de paroi universellement admises :

- sous-couche visqueuse

$$
0<y^{+}<6
$$

- zone intermédiaire

$$
6<y^{+}<35
$$

- zone logarithmique

$$
35<y^{+}<150 \Rightarrow \frac{y}{H} \leqq 0,05 .
$$

Précisons que les points expérimentaux correspondant à la sous-couche visqueuse ont été corrigés en tenant compte de l'influence de la paroi sur les mesures au fil chaud $(33,34)$.

La loi de paroi $u^{+}=y^{+}$est bien vérifiée pour $\left(y^{+}<10\right)$. Le profil reste inchangé lorsque la fréquence de perturbation imposée varie.

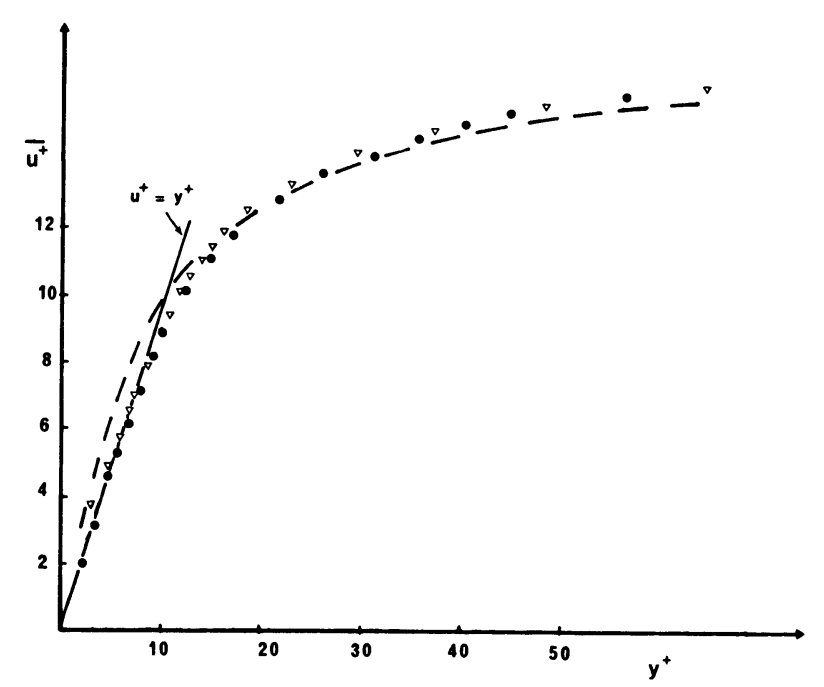

Fig. 4. - Profil de la vitesse moyenne près de la paroi : - Tartarin; écoulement instationnaire (quelle que soit la fréquence) $R e=31300 ; \nabla$ Hussain et Reynolds [12]; écoulement instationnaire, $R e=32300$; - . - Comte-Bellot [32]; écoulement stationnaire, $R e=120000$.

[Mean velocity profile near the wall : Tartarin; unsteady flow, $R e=31300$ (any frequency); $\nabla$ Hussain et Reynolds [12]; unsteady flow, $R e=32300$; - . - ComteBellot [32]; steady flow, $R e=120000$.]

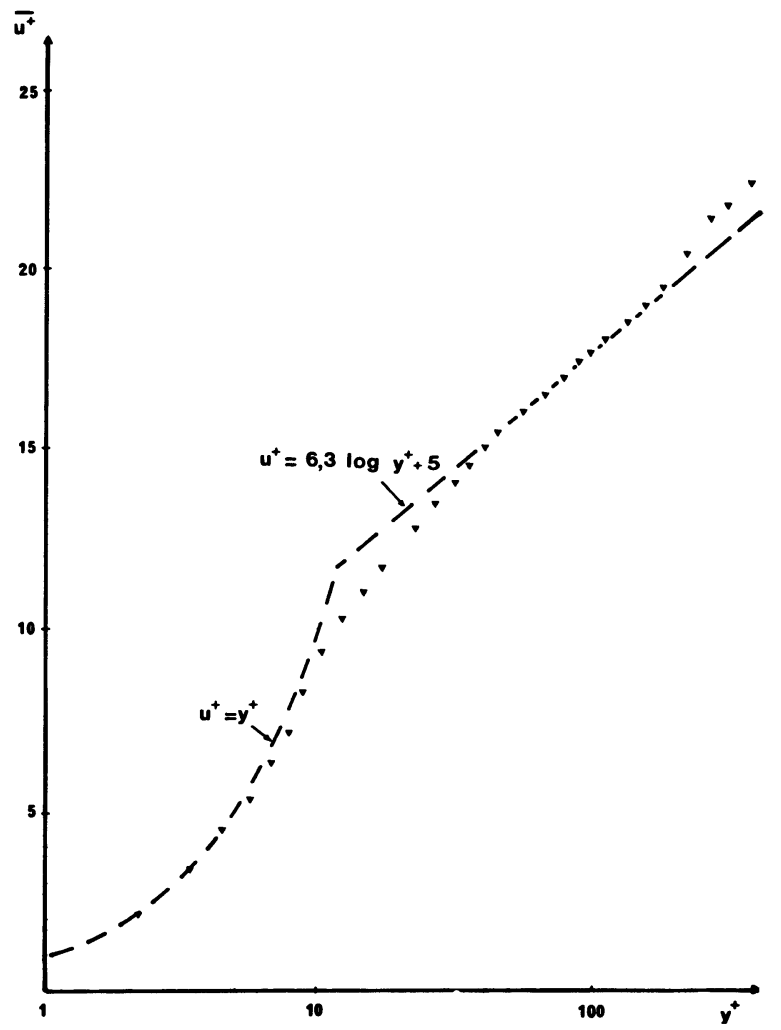

Fig. 5. - Profil de la vitesse moyenne : représentation logarithmique; écoulement instationnaire (pour toutes les fréquences étudiées).

[Mean velocity profile : logarithmic scale; unsteady flow for all frequencies tested.]

5.4 La figure 6 montre le profil de la fluctuation turbulente $\sqrt{{u^{\prime 2}}^{\prime 2}}$ semblable à ceux rencontrés dans la littérature (à titre d'exemple nous avons comparé ce profil à celui rapporté par G. Comte-Bellot dans le cas d'un écoulement stationnaire à nombre de Reynolds proche du nôtre). Ici également, les résultats concernant la sous-couche visqueuse $\left(y^{+}<6\right)$ ont été corrigés en tenant compte de l'influence de la paroi. Notons une bonne concordance avec la droite $\sqrt{{\text { u}^{\prime 2}}^{2}}=0,30 u_{\tau} y^{+}$ proposée par Laufer (pour $y^{+} \rightarrow 0$ ) à la suite de mesures en conduit cylindrique (cf. [28]). Le profil du facteur de dissymétrie $\left(S=\overline{u^{\prime 3}} /\left(\overline{u^{\prime 2}}\right)^{3 / 2}\right)$ de la composante turbulente de la vitesse (Fig. 6) n'est pas modifié par l'instationnarité de l'écoulement, et est cohérent avec les résultats concernant les zones où prédominent l'apport de fluides $\left(u^{\prime}>0, y^{+}<15\right)$ et l'éjection $\left(u^{\prime}<0, y^{+}>15\right)$.

Les figures 2 à 6 montrent que le fait d'imposer une perturbation périodique de faible amplitude et de fréquence variable proche de la fréquence caractéristique $N_{\mathrm{b}}$ ne modifie aucunement les caractéristiques " en moyenne" d'un écoulement turbulent. Cette constatation n'est pas nouvelle $[13,14,23]$ et était prévisible à la suite des travaux théoriques de Binder et Kuency [14], compte tenu de nos conditions expérimentales. Nous y reviendrons. 


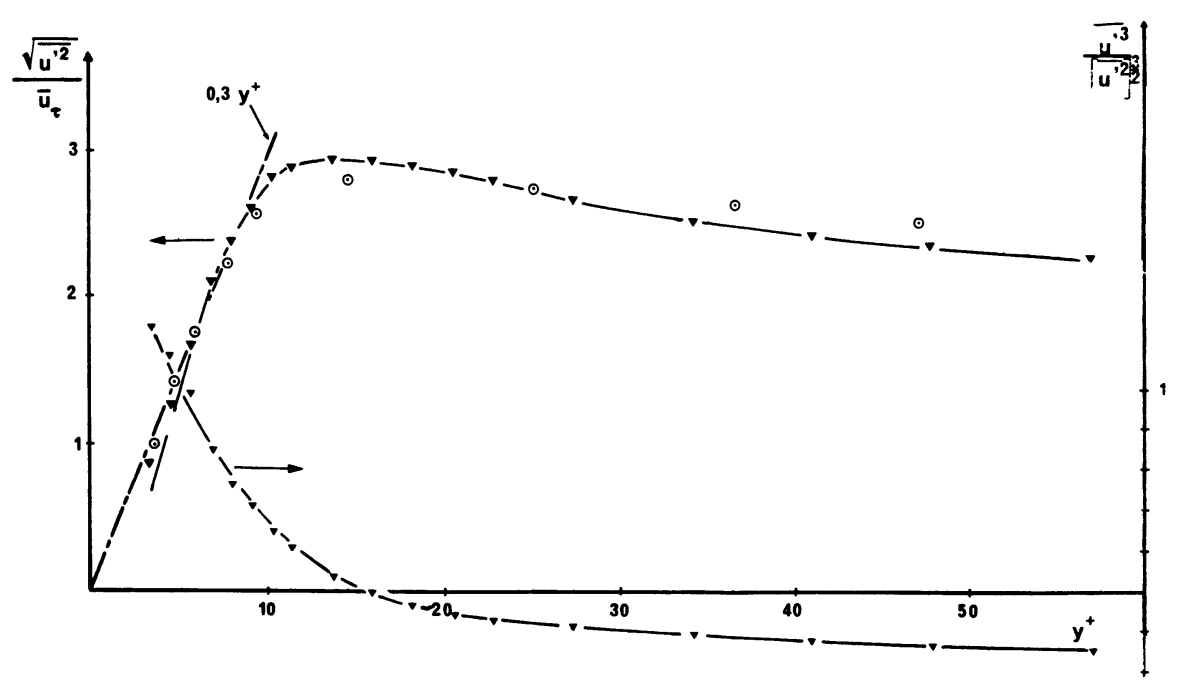

Fig. 6. - Profil de l'intensité de turbulence moyenne et du facteur de dissymétrie (pour toutes les fréquences étudiées). $\nabla$ Tartarin; écoulement instationnaire, $R e=31300 ; \odot$ Comte-Bellot; écoulement stationnaire [32], $R e=57000$.

[Mean turbulent intensity profile near the wall and skewness factor. $\nabla$ Tartarin; unsteady flow for all frequencies tested, $R e=31300 ; \odot$ Comte-Bellot; steady flow [32], $R e=57$ 000.]

5.5 ProfiLS de LA CONTRIBUTION PÉRIODIQUe $|\tilde{u}| .-$ La figure 7 fait apparaître un paramètre important qui est la fréquence de la perturbation imposée. Bien que nous n'ayons pas représenté l'ensemble des courbes obtenues pour clarifier la figure, deux constatations importantes sont à retenir; pour les fréquences imposées supérieures à $N_{\mathrm{b}}$ (i.e. $N=26 \mathrm{~Hz}$ et $N=$ $29 \mathrm{~Hz}$ ) apparaît une amplification notable de la contribution périodique $|\tilde{u}|$ près de la paroi : le "dépassement " atteint $70 \%$, tandis qu'à titre de comparaison celui-ci n'est que de l'ordre de $7 \%$ dans le cas d'un écoulement laminaire instationnaire [13]. Pour les fréquences inférieures à $N_{\mathrm{b}}$ (i.e. : $N=24 \mathrm{~Hz}$ et $N=22 \mathrm{~Hz}$ ) les profils révèlent une évolution plus inattendue : une décroissance brutale de l'amplitude $|\tilde{u}|$ se produit lorsque : $7<y^{+}<12$ (en particulier pour $N=22 \mathrm{~Hz}$ ). Nous sommes là en présence d'un phénomène à souligner car il a déjà été rencontré par Acharya et Reynolds [13] dans des conditions expérimentales proches des nôtres et par Binder et Kueny [14,

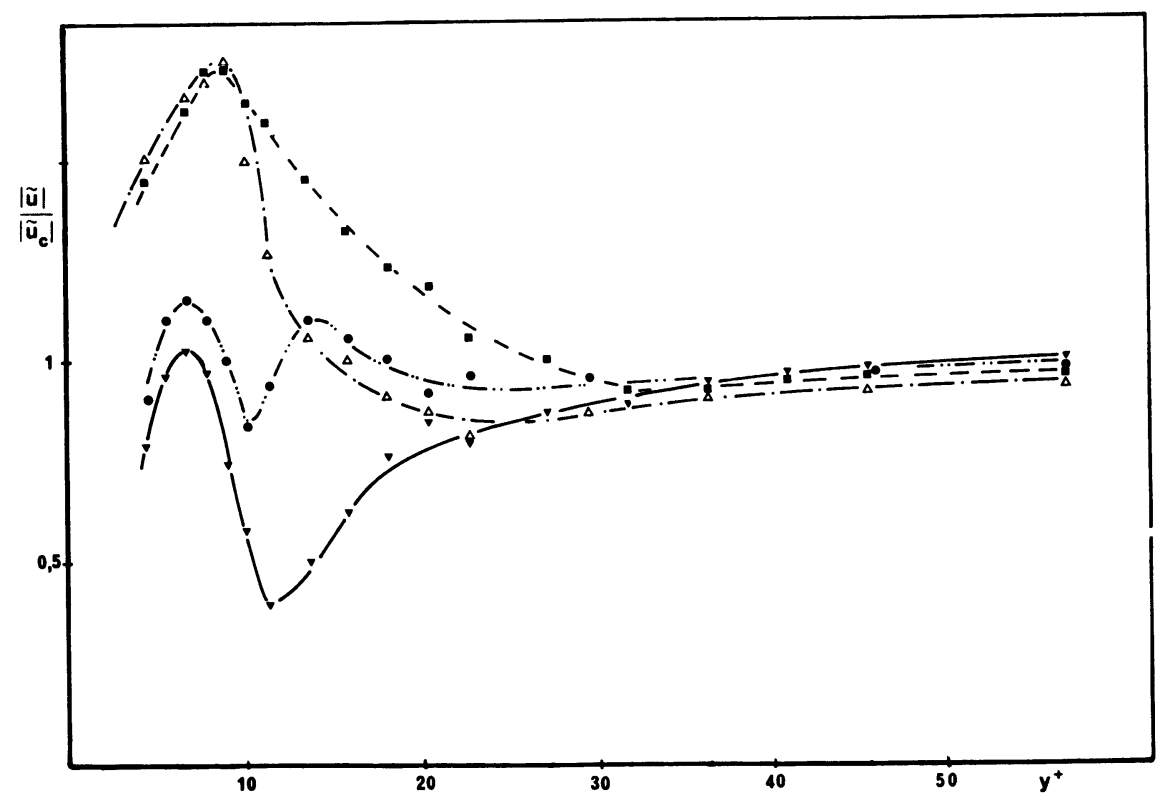

Fig. 7. - Profil de l'amplitude de la composante périodique de la vitesse. $\nabla N=22 \mathrm{~Hz}, \bullet N=24 \mathrm{~Hz}, \Delta N=26 \mathrm{~Hz}$, $N=29 \mathrm{~Hz}$

[Profile of the periodic velocity oscillation amplitude.] 


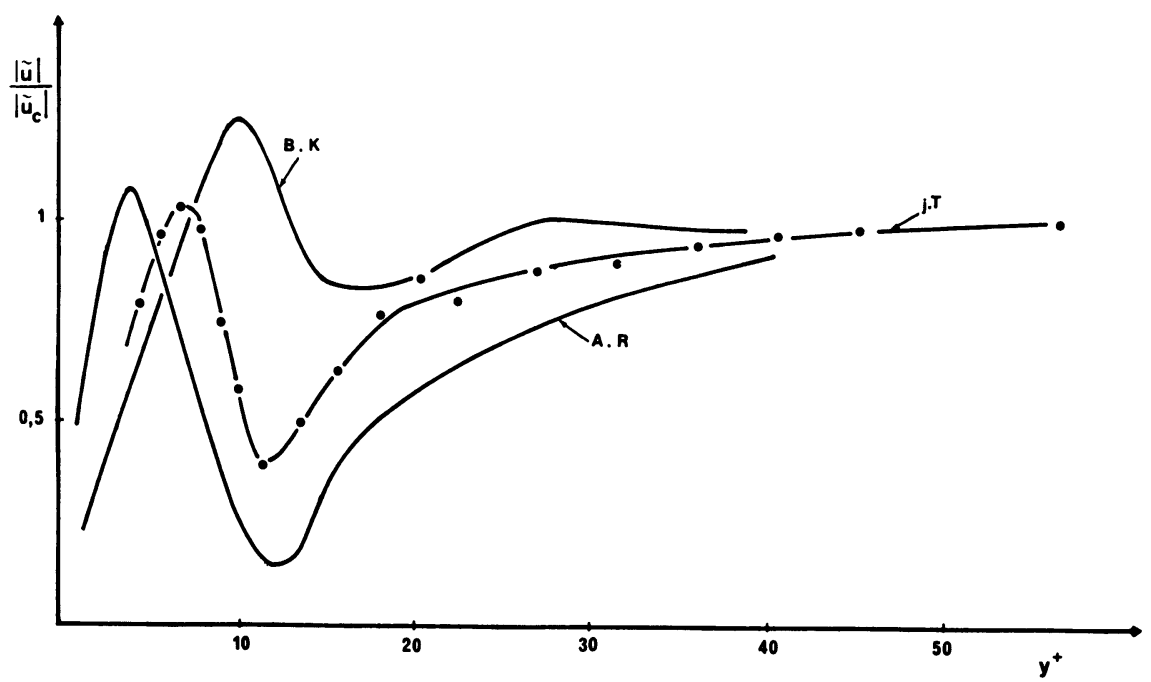

Fig. 8. - Comparaison des profils de la composante périodique de la vitesse obtenus par : A.R. : Acharya et Reynolds [13], pour $N / N_{\mathrm{b}}=0,96$ et $R e=13800$, B.K. : Binder et Kueny [27], pour $N / N_{\mathrm{b}}=0,97$ et $R e=88000$, j.T. : Tartarin, pour $N / N_{\mathrm{b}}=0,88$ et $\operatorname{Re}=31300$.

[Periodic velocity component profiles from : A.R. Acharya et Reynolds [13], for $N / N_{\mathrm{b}}=0,96$ et $\operatorname{Re}=13800$, B.K. : Binder et Kueny [27], for $N / N_{\mathrm{b}}=0.97$ et $R e=88000$, j.T. : Tartarin, for $N / N_{\mathrm{b}}=0.88$ et $R e=31300$.]

27] dans un canal hydraulique (bien que, pour ces derniers, le phénomène semble plus atténué). Or, c'est précisément et uniquement pour des fréquences proches de la fréquence $N_{\mathrm{b}}$ (Fig. 8) que le phénomène a été observé : $N / N_{\mathrm{b}}=0,97$ chez Acharya et Reynolds [13], $N / N_{\mathrm{b}}=0,96 \mathrm{chez}$ Binder et Kueny $[14,27]$ et $N / N_{\mathrm{b}}=0,88$ dans notre cas (en précisant que cette -valeur correspond à l'observation " optimale " de la décroissance brutale de $|\tilde{u}|)$. Nous reviendrons en conclusion sur une interprétation plausible de ce phénomène.

5.6 Nous présentons (Fig. 9) le déphasage de la composante périodique de vitesse pour la fréquence $N=22 \mathrm{~Hz}$. L'origine de la phase étant prise au mini- mum de vitesse périodique sur l'axe de la soufflerie nous constatons (comme Acharya et Reynolds [13]) un retard de phase lorsque nous approchons de la paroi. Précisons que l'incertitude absolue sur la détermination du déphasage est de l'ordre de $15^{\circ}$, pour la fréquence présentée ici. Pour les autres fréquences l'évolution du déphasage est semblable, mais compte tenu d'une plus grande dispersion expérimentale nous ne donnons que la courbe la plus crédible (hormis le premier point à $y^{+}=3,5$ ).

5.7 Les courbes de la figure 10 montrent l'évolution du profil de la contribution périodique $\sqrt{\left|\tilde{r}_{11}\right|}$ aux fluctuations turbulentes. Nous observons que la réponse de la turbulence à la perturbation imposée

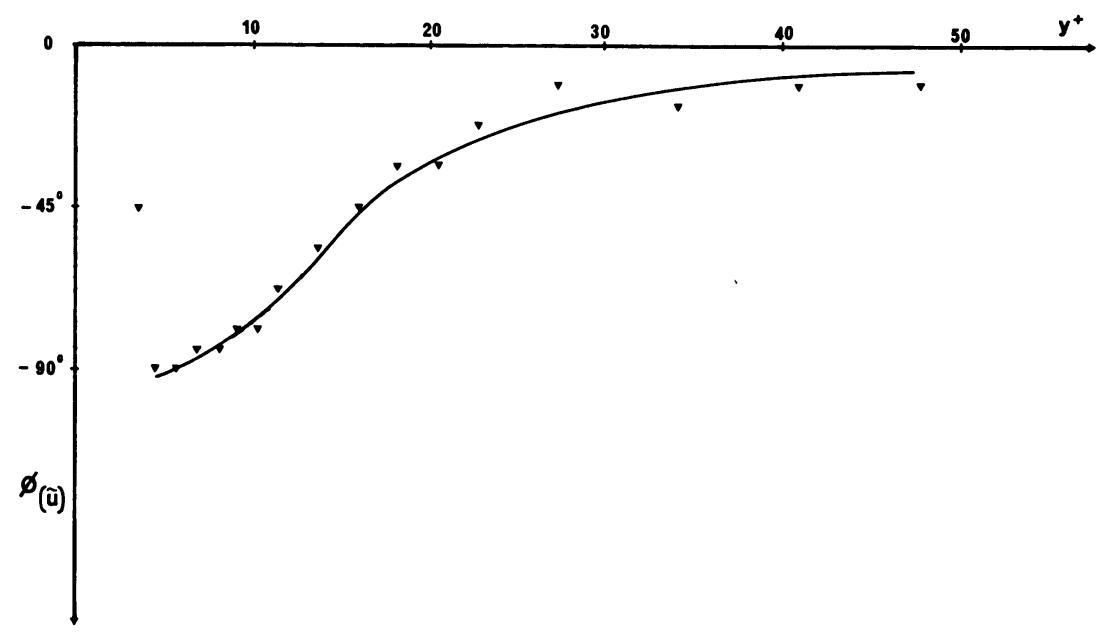

Fig. 9. - Déphasage de la composante périodique de vitesse $(N=22 \mathrm{~Hz})$.

[Phase of the periodic velocity oscillation $(N=22 \mathrm{~Hz})$.] 


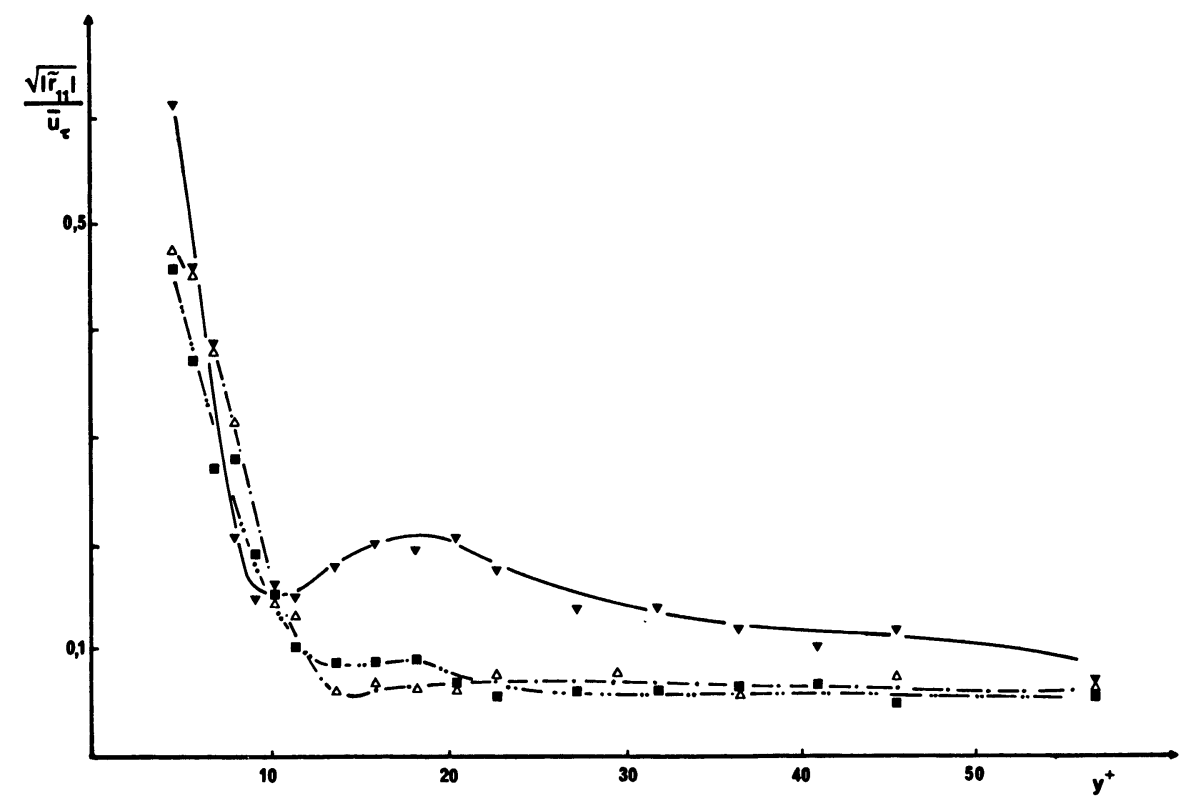

Fig. 10. - Profil de l'amplitude de la fluctuation périodique de l'intensité de turbulence : $\nabla N=22 \mathrm{~Hz}, \boldsymbol{\square}=26 \mathrm{~Hz}$, $\Delta N=29 \mathrm{~Hz}$.

[Profile of the amplitude of the turbulent intensity periodic oscillations.]

est très amplifiée près de la paroi $\left(5<y^{+}<10\right)$. Précisons cependant que les vibrations inévitables de la structure de la soufflerie sont cause d'incertitudes sur les valeurs absolues obtenues très près de la paroi $\left(y^{+}<6\right)$, sans parler des erreurs liées à l'anémométrie dans ce domaine. Au-delà, nous avons vérifié en rigidifiant plus ou moins les plaques planes de la soufflerie, la reproductibilité des résultats expérimentaux.
Les valeurs relatives de la grandeur $\sqrt{\left|\tilde{r}_{11}\right|}$ présentées à la figure 11 sont identifiables à des taux de turbulence " alternatifs ». Nous observons qu'ils sont d'un ordre de grandeur deux fois supérieur aux taux « moyens » $\left(\sqrt{{u^{\prime 2}}^{2}} \sqrt{u}\right)$. Le résultat le plus notable est de constater qu'au minimum " relatif » de la contribution périodique $|\tilde{u}|$ observé (Fig. 8) pour la fréquence $22 \mathrm{~Hz}$ correspond ici un maximum relatif du taux de turbulence à la distance $y^{+} \cong 12$.

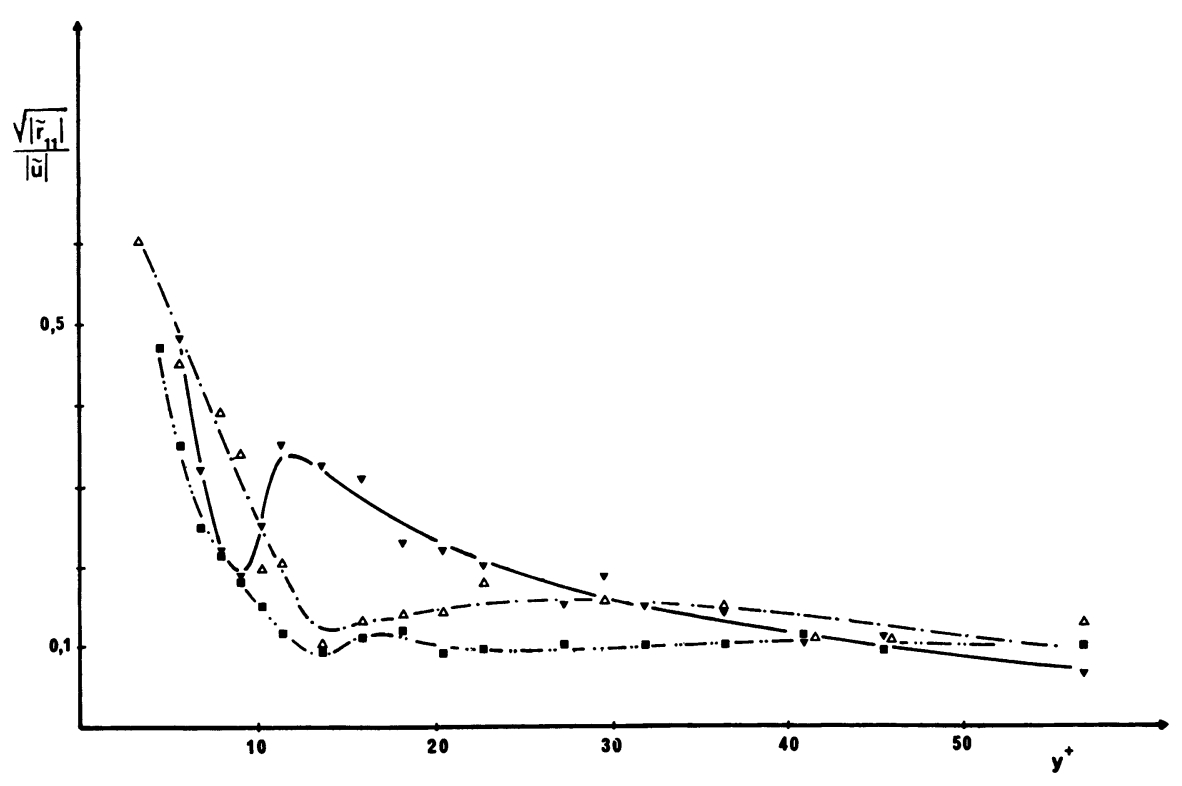

Fig. 11. - Profil de l'amplitude de la fluctuation périodique du taux de turbulence : $\nabla N=22 \mathrm{~Hz}, \square N=26 \mathrm{~Hz}, \Delta N=$ $29 \mathrm{~Hz}$.

[Profile of the relative amplitude of turbulent intensity periodic oscillations.] 


\section{Discussion.}

En écoulement instationnaire, la zone de paroi est le domaine de phénomènes notables sur lesquels il est nécessaire de revenir. Cette zone pariétale est en effet le siège de gradients de vitesse importants $(\partial \bar{u} / \partial y$, $\partial \tilde{u} / \partial y$ par exemple). Comme l'ont souligné Binder et Kueny [14], la turbulence ne peut être affectée par l'oscillation du champ de vitesse, mais elle peut l'être par l'existence de ces gradients.

En présence d'une perturbation périodique de faible amplitude et quelle que soit sa fréquence, la turbulence ne serait donc modifiée notablement par rapport à son état «stationnaire" que si le gradient de vitesse " alternative » $(\partial|\tilde{u}| / \partial y)$ était de l'ordre de grandeur du gradient de vitesse « moyenne » $(\partial \bar{u} / \partial y)$. La plupart des travaux expérimentaux rapportés $[12,13,14,25$, $26, \ldots$.$] montre que dans le cas général la turbulence$ n'est effectivement pas ou peu altérée. En effet, il y a apparemment incompatibilité entre le fait d'imposer une perturbation de faible amplitude $(|\tilde{u}| / \tilde{u} \ll 1)$ et d'obtenir des gradients $(\partial|\tilde{u}| / \partial y)$ et $(\partial \bar{u} / \partial y)$ de même ordre de grandeur.

Cependant, le phénomène illustré par la figure 9 montre l'existence d'un gradient $\partial|\tilde{u}| / \partial y$ antagoniste du gradient $\partial \bar{u} / \partial y$. Bien que les ordres de grandeurs de ces gradients ne soient pas semblables (le rapport des deux étant de l'ordre de $5 \%$ ), ils sont de signe opposé dans une zone étroite $\left(7<y^{+}<15\right)$ mais primordiale quant à la phénoménologie de la turbulence. On constate de façon concomittante, une amplification notable de "l'oscillation» $\sqrt{\left|\tilde{r}_{11}\right|}$ de la fluctuation turbulente (cf. Figs. 10 et 11).

Rappelons que ce domaine spatial $\left(7<y^{+}<15\right)$ est le lieu privilégié d'apparition des bouffées turbulentes telles qu'elles ont été observées expérimentalement en écoulement stationnaire (cf. par exemple [6, 7, $8, \ldots])$. Cette coïncidence, qui ne semble pas fortuite, nous permet d'avancer une hypothèse prenant en compte le caractère alternatif de l'écoulement : le débit pulsé entraîne lors de chaque cycle une phase accélératrice du champ de vitesse puis une phase décélératrice. Nous pouvons donc concevoir qu'au cours d'une période sont respectivement favorisées les séquences "apport» de fluides et «éjection", rappelées précédemment, en particulier lorsque les fréquences de perturbation et d'apparition des bouffées turbulentes coïncident. Nous pensons que la perturbation imposée « entretient » le processus et que localement $\left(10<y^{+}<30\right)$ la structure de la turbulence est modifiée; le champ de vitesse périodique est altérée : une partie de l'énergie liée à la composante alternative $(\tilde{u})$ est transférée à l'oscillation de la turbulence $\left(\tilde{r}_{11}\right)$, sans que cela ne modifie sensiblement le champ moyen (cf. Figs. 4, 5, 6). L'équation 12 montre la dépendance des termes " $\tilde{r}_{11}$ " et $" \tilde{u}$ » et rend plausible l'hypothèse d'un transfert d'énergie. Il convient de rester prudent en abordant ici la phénoménologie fondamentale de la turbulence encore peu connue dans cette zone proche de la paroi (a fortiori, en écoulement instationnaire).

\section{Conclusion.}

La fréquence de perturbation imposée à un écoulement turbulent est un paramètre essentiel, lorsqu'elle est de l'ordre de grandeur de la fréquence caractéristique d'apparition des bouffées turbulentes $\left(N_{\mathrm{b}}\right)$. Notre étude expérimentale et la confrontation de nos résultats à ceux existant dans la littérature montrent que la zone pariétale où interfèrent la couche de Stokes et la zone intermédiaire $\left(5<y^{+}<25\right)$ est alors le siège de comportements singuliers du champ de vitesse " périodique ". Les phénomènes observés et l'apparition des bouffées turbulentes sont, semble-til, très liés : le processus, intermittent en stationnaire, étant « entretenu " par la perturbation imposée pourrait expliquer les transferts d'énergie constatés, la turbulence étant très localement modifiée. C'est cet aspect phénoménologique que nous devrons examiner dans l'avenir.

\section{Bibliographie}

[1] Blackwelder, R. F., Coherent structures in turbulent boundary layers, Conf. proc. A.G.A.R.D. $\mathrm{n}^{\circ} 271$ (1980) 24.1 à 24.6.

[2] ANDrew, S. W., Thomas, Some observations of the structure of the turbulent boundary layer. Conf. proc. A.G.A.R.D. no 271 (1980) 26.1 à 26.11.

[3] Nakagawa, H. and Nezu, I., Structure of space-time correlations of bursting phenomena in an openchannel flow. J. Fluid Mech. 104 (1981) 1 à 43.

[4] Van MaAnen, H. R. E., Experimental study of coherent structures in the turbulent boundary layer of pipe flow using laser-doppler anemometry. Conf. proc. A.G.A.R.D. no 271 (1980) 3.1 à 3.20.
[5] Laufer, J. and Narayanan, N. B., Mean period of the turbulent production mechanism in a boundary layer. Phys. Fluids 14 (1971) 182 à 183.

[6] SAвот, J. and Cомте-Bellot, G., Intermittency of coherent structures in the core region of fully developed turbulent pipe flow. J. Fluid Mech. 744 (1971) 767 à 796.

[7] RaO, K. N., Narasimha, R. and Narayanan, M. A., The bursting phenomenon in a turbulent boundary layer. J. Fluid Mech. 482 (1971) 339 à 352.

[8] Elena, M., Fulachier, L. and Dumas, R., Etude expérimentale des apports et éjections de fluide dans une couche limite turbulente. Conf. proc. A.G.A.R.D. no 271 (1980) 2.1 à 2.21 . 
[9] BlaCkWElder, R. F. and Kaplan, R. E., On the wall structure of the turbulent boundary layer. J. Fluid Mech. 761 (1976) 89 à 112.

[10] Antonia, R. A., Danh, H. Q. and Prabhu, A., Bursts in turbulent shear flows. Phys. Fluids 1911 (1976) 1680 à 1686.

[11] Davies, P. O. A. L. and Yule, A. J., Coherent structures in turbulence. J. Fluid Mech. 693 (1975) 513 à 537.

[12] Hussain, A. K. M. F. and ReYnolds, W. C., The mechanics of a perturbation wave in turbulent shear flow. Report F.M. 6, Stanford University (1970).

[13] Acharya, M. and ReYnolds, W. C., Measurements and prediction of a fully developed turbulent channel flow with imposed controlled oscillations. Report T.F. 8, Stanford University (1975).

[14] Binder, G. and KuENY, J. L., Measurements of the periodic velocity oscillations near the wall in unsteady turbulent channel flow. Third Symposium on " Turbulent shear flow ", Davis (1981) 8.19 à 8.25.

[15] Ramaprian, B. R. and ShUEN-Wei, TU, An experimental study of oscillators pipe flow at transitional Reynolds numbers. J. Fluid Mech. 1003 (1980) 513 à 544.

[16] Kita, Y., Adachi, Y. and Hirose, K., Periodically oscillating turbulent flow in a pipe. Bull. J.S.M.E. 23179 (1980) 656 à 664.

[17] SHEMER, L. and WygnNANSKI, I., On the pulsating flow in a pipe. Third Symposium on "Turbulent shear flow ", Davis (1981).

[18] NoRRIS, H. L. and REYNOLDS, W. C., Turbulent channel flow with a moving wavy boundary. Report T.F. 7 Stanford University (1975).

[19] Houdeville, R., Desopper, A. and Cousteix, J., Analyse expérimentale des caractéristiques d'une couche limite turbulente en écoulement pulsé. Essai de prévision théorique. La Rech. Aérosp. 4 (1976) 183 à 191.

[20] Cousteix, J., Houdeville, R. and JAVelle, J., Response of a turbulent boundary layer to a pulsation of the external flow with and without adverse pressure gradient. In " Unsteady turbulent shear flows", I.U.T.A.M. Symposium, Toulouse (1981).

[21] Patel, M. H., On turbulent boundary layers in oscillatory flow. Proc. Roy. Soc. A 353 (1977) 121 à 144.

[22] CousteIx, J., Couches limites tridimensionnelles ou instationnaires. Présenté au « 18 e colloque d'Aérodynamique Appliquée "; Poitiers (1982).
[23] Parikh, P. G., Jayaraman, R. and Reynolds, W. C., Dynamics of an unsteady turbulent boundary layer. Third Symposium on "Turbulent shear flow ", Davis (1981).

[24] Hayashi, T. and OHASHI, M., A dynamical and visual study on the oscillatory turbulent boundary layer. Third Symposium on "Turbulent shear flow ", Davis (1981).

[25] Ronnenberger, D. and Ahrens, C. D., Wall shear stress caused by small amplitude perturbation of turbulent boundary layer flow : an experimental investigation. J. Fluid Mech. 833 (1977) 433 à 464.

[26] CARR, L. W., A review of unsteady turbulent boundary layer experiments I.U.T.A.M. Symposium on "Unsteady turbulent shear flows " Toulouse (1981).

[27] Binder, G. and KuENY, J. L., Measurements of the periodic velocity oscillations near the wall in unsteady turbulent channel flow. I.U.T.A.M. Symposium on "Unsteady turbulent shear flows " Toulouse (1981).

Ouvrages généraux

[28] Favre, Kovasnay, Dumas, Gaviglio, Coantic, La turbulence en Mécanique des Fluides (Gauthier-Villars éditeurs).

[29] Hinze, Turbulence (Mc Graw-Hill éditeurs).

[30] Michel, Cousteix, Houdeville, Unsteady Turbulent shear flows. I.U.T.A.M. Symposium, Toulouse (Springer-Verlag éditeurs) 1981.

[31] MAYE, J. P., Contribution à une étude statistique des champs thermiques turbulents. Thèse présentée à l'Université de Poitiers (1976).

[32] Comte-Bellot, G., Ecoulement turbulent entre deux parois parallèles. Publications scientifiques du Ministère de l'Air (1965) no 419.

[33] HebBaR, K. S., Wall proximity correction for hot-wire readings in turbulent flows. Disa Information $\mathrm{n}^{\circ} 25$ (1980).

[34] Chauve, M. P., Détermination des contraintes de frottement à la paroi par anémométrie fil chaud. Compte rendu « Euromech 132 » (« Hot-wire, hot film, anemometry and conditional measurements "). Lyon, 1980.

[35] Promode, R. Bandyopadhyay, Period between bursting in turbulent boundary layers. Phys. Fluids 2510 (1982). 